\title{
Um novo bebê: interpretações sobre competências
}

\author{
Ana Cristina Bortoletto-Dunker \\ Eulina da Rocha Lordelo
}

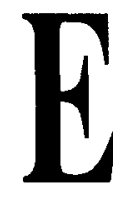

ste artigo pretende atualizar nossa visão sobre o recém-nascido, à luz dos novos conhecimentos produzidos na pesquisa sobre as capacidades do bebê, a partir de uma nova perspectiva.

Até a década de 50, era uma crença corrente entre os profissionais de saúde, a idéia de que os bebês não podiam ver, ouvir e se comunicar, sendo o mundo exterior confundido com o seu próprio corpo; seus movimentos eram vistos como aleatórios ou como produtos de seus reflexos desorganizados. $\mathrm{O}$ recémnascido e o bebê eram vistos como organismos basicamente deficientes, incompletos e relativamente incompetentes e inadequados (Thoman, 1979). Seu equipamento sensorial e perceptivo eram tidos como rudimentares. Suas experiências teriam pouco significado para sua vida futura, devido a ausência de memória; o ser humano nasceria como uma página em branco, em termos de emoções, temperamento, preferências; a criança seria um ser a-social, que aprenderia os sentimentos "humanos" como amor, culpa e ódio, por exemplo, como resultado das associações presentes no curso do desenvolvimento, entre outras crenças, mais ou menos "fundamentadas" cientificamente.

Uma visão particularmente estranha- da no "clima" intelectual dessa época, e que ainda persiste parcialmente nos nossos dias é o que Thoman chamou "infância como período de promessa". Muito da pesquisa realizada até então visava relacionar o comportamento do bebê ao seu desenvolvimento posterior, com base na suposição de que técnicas mais refinadas de avaliação permitiriam prever 0 curso do desenvolvimento, identificando fatores de risco para a vida normal da criança e do adulto.

Nos últimos 30 anos, assistimos a uma profunda transformação no modo de ver o recém-nascido, tanto numa vertente humanista, identificada como uma preocupação com o respeito à criança como ser humano, como nos conhecimentos produzidos pela pesquisa, que vem revolucionando as crenças sobre as capacidades e especificidades do recémnascido.

Uma das mais notáveis revisōes na área é a que deslocou o interesse pelo bebê como "o pai do adulto" para o bebê em si, sendo parte dessa transformação de responsabilidade da Etologia, que propôs a infância como um período de vida suficientemente importante para justificar adaptações específicas que maximizassem as chances de sobrevivência do indivíduo. Essa mudança, aparentemente sutil, teve implicações pro- 
fundas no modo de "ver" os fenômenos da infância, como pode ser ilustrado no exemplo do fenômeno do apego. Os fortes vinculos que se estabelecem entre o bebê e a figura principal de cuidado, geralmente a mãe, foram vistos por muitas gerações como sinal de dependência, um sintoma que deveria ser combatido, tendo em vista sua não funcionalidade para a vida adulta. O deslocamento do foco de interesse para a própria infância, como periodo importante em si mesmo, ressignificou o fenômeno, evidenciando seu valor de si mesmo, ressignificou o fenômeno, evidenciando seu valor de sobrevivência para a criança. Outros exemplos dessa mudança de enfoque são o choro, o brinquedo e as relações entre crianças.

Por outro lado, os avanços no conhecimento das suas capacidades e habilidades foram essenciais para um aumento da nossa compreensão sobre os bebês, particularmente os recém-nascidos. Essas descobertas cobrem os campos sensorial, perceptivo e afetivo e dãoà luz um ser altamente complexo e organizado, preparado para a sobrevivência nas condições da espécie, apto e disponível para a construção de trocas e relações sociais, como pode ser visto no breve resumo apresentado a seguir, focalizando duas grandes áreas de estudo:

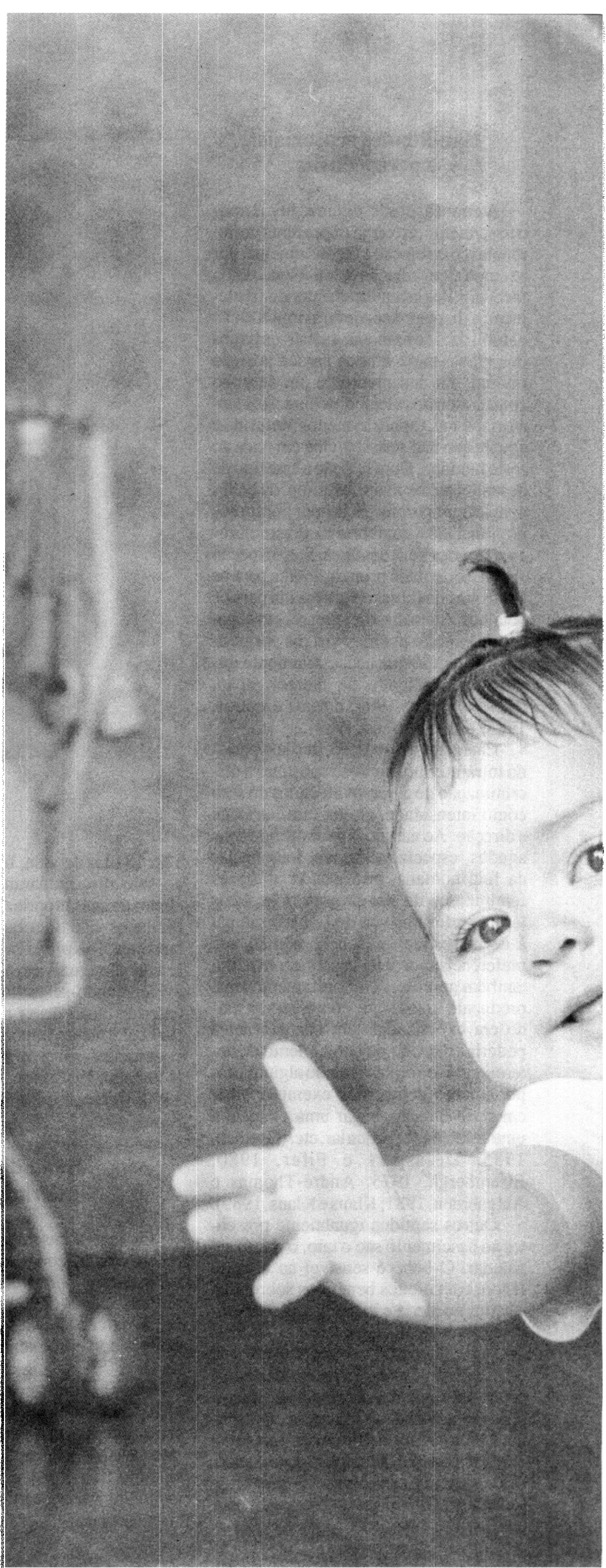




\section{Habilidades sensoriais e perspectivas}

A criança, desde algumas horas após o nascimento, enxerga e apresenta preferências com relação a alguns objetos; por exemplo, em relação a um esquema da face, privilegia aqueles contendo os elementos dispostos segundo a organização natural da face humana, a saber, os traços dos olhos, nariz e boca na sua posição normal, em detrimento de um desenho com os mesmos elementos dispostos aleatoriamente. Opadrão menos preferidoé aquele que não se assemelha em nada ao rosto humano. Com relação a esse tipo de desenho, até os mais abstratos, os bebês apresentam poreferências por contornos pronunciados, com forte contraste claroescuro e ricos em detalhes. Sua inspeção visual é orientada primeiramente para os contornos da figura e em seguida para os detalhes. Além disso, eles parecem capazes de guardar padrões visuais por periodos de tempo que necessariamente implicam capacidade de memorização. (Field e outros, 1984; e Masi e outros, 1983; Klaus e Klaus, 1989).

Em relação à audição, desde o período intra-uterino, pode-se constatar a discriminação de diversos atributos do som como intensidade, altura, familiaridade e direção. Ao nascer, o bebê prefere sons agudos, especialmente nas frequências da fala humana, priorizando as vozes femininas e da sua própria mãe. Mais tarde, preferirá a voz do seu próprio pai à de um estranho. $O$ bebê não só tem preferências por determinados sons como também apresenta comportamentos que produzam esses sons, como demonstrado em experiências em que a criança poderia controlar o aparecimento de determinado som apresentando algum comportamento como, por exemplo olhar em certa direção, sugar uma chupeta a uma velocidade particular, etc. (Gottlieb, 1985; Decaspers e Fifer, 1980; Eisembergh, 1975; André-Thomas e Autgaerden, 1981; Klaus e Klaus, 1989).

Outros sentidos igualmente presentes ao nascimento são o tato, o olfato e o paladar. $O$ bebê é sensível ao contato físico, com efeitos benéficos sobre seus estados de alerta e inquietação. Respondem também a diferenças de textura, umidade, temperatura e pressão, além de estímulos dolorosos. O recém-nascido é capaz de discriminar pequenas alteraçōes nas substâncias quimicas, apresentando preferências por certos sabores e odores (em geral, adocicados contra azedos e amargos) com respostas de expressões faciais de prazer e desprazer. Com

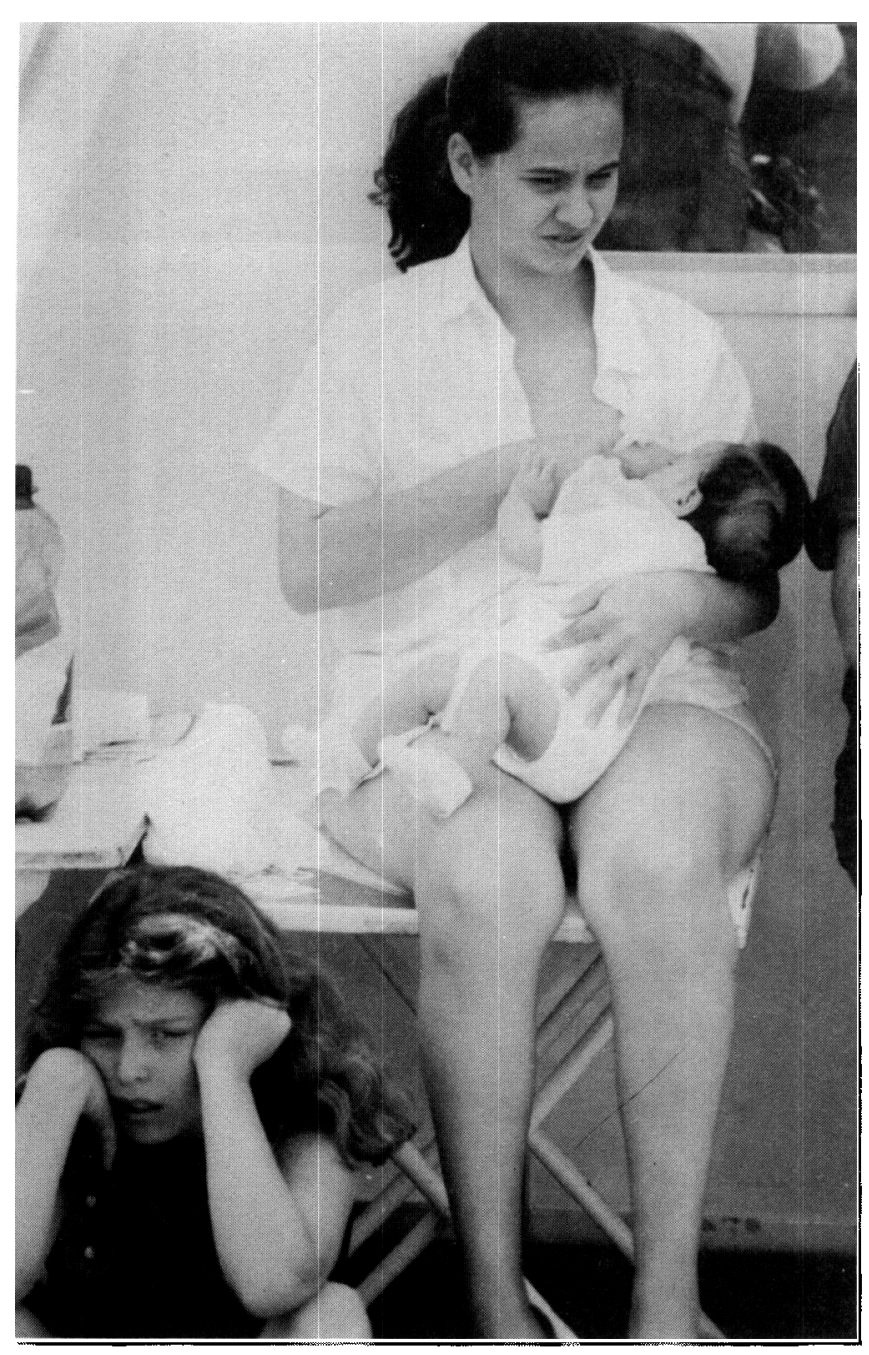

12 a 18 dias de vida, bebês alimentados ao seio discriminaram e preferiram o cheiro de suas próprias mães, contra o de mulheres não familiares, mesmo que lactantes. Tais preferências absolutamente não são iguais em todas as crianças, evidenciando-se estilos individuais de exteriorização dessa reações, um dado particularmente importante para nossas concepções sobre práticas de criação. (Montagu, 1988; Bergamasco e Beraldo 1990; Cemoch e Porter, 1985; Klaus e Klaus, 1989).

\section{Habilidades interativas}

Os recém-nascidos apresentam um repertório de comportamentos que os capacitam para as trocas sociais com o seu meio, através de sinais comunicativos e de capacidades de regular o seu próprio comportamento pelo do parceiro.

Os bebês discriminam diferentes expressð̋es faciais de emoç̃es básicas, como alegria, surpresa e tristeza. Aos três meses, $o$ bebê apresenta um conhecimento complexo dessas expressōes, incluindo variações de uma expressão numa mesma categoria; por exemplo, eles percebem diferentes intensidades de sorriso como uma mesma expressão facial, mas são capazes de discriminar entre elas. Além de discriminar, o bebê produz todas as expressões faciais básicas conforme a categorizaçāo de Eckman (1982), consideradas universais na espécie humana. Por exemplo, algumas de suas reações a cheiros, sabores, estímulos visuais, estímulos dolorosos incluem expressőes faciais indicadoreas de prazer e desprazer (Kuchuk e outros, 1986; Nelson, 1987).

Uma habilidade particularmente intrigante, objeto de intermináveis discussões na área e a imitação de expressões faciais, a partir de poucas horas de vida) Diante de um modelo posando expressões de tristeza, surpresa e alegria, os bebês reproduzem as mesmas expresões, reconheciveis por observadores inconscientes da expressão que está sendo apresentada pelo modelo (Field e outros, 1982; Meltzoff e Moore, 1977 e 1985; Vinter, 1987).

Todas essas habilidades estão, aparentemente, a serviço das funçðes comunicativas, de forma organizada e integrada. Uma qualidade definidora do com- 
"As capacidades agora conhecidas do bebê são surpreendentes, mesmo

espantosas, em

comparação com as

crenças anteriores de que

o bebê nascia

praticamente cego. Como integrá-las numa visão

compreensiva? Afinal, a que vêm essas capacidades?" portamento do bebê e da mãe é o que se denomina sincronia ou co-regulação, que implica pensar os comportamentos da díade como um sistema mutuamente adaptado. Vários estudos utilizando técnicas de micro-análise de filmagens da interação mãe-bebê demonstraram que o ritmo de movimento do bebê corresponde ao ritmo empregado na fala da mãe, que as mães ajustam sua fala, em termos de tons, altura e ritmo às respostas do bebê, numa configuração descrita como coregulação, ou seja, os comportamentos do bebê e da mãe afetam os comportamentos do outro, de modo dinâmico e complexo. (Trevarthen, 1989; Papousek e Papousek, 1989; Koester e outros, 1989).

\section{Para compreender esse brilhante recém-nascido}

As capacidades agora conhecidas do bebê são surpreendentes, mesmo espantosas, em comparação com as crenças anteriores de que o bebê nascia praticamente cego. Como integrá-las numa visão compreensiva? Afinal, a que vêm essas capacidades?

A abordagem etológica ao estudo do

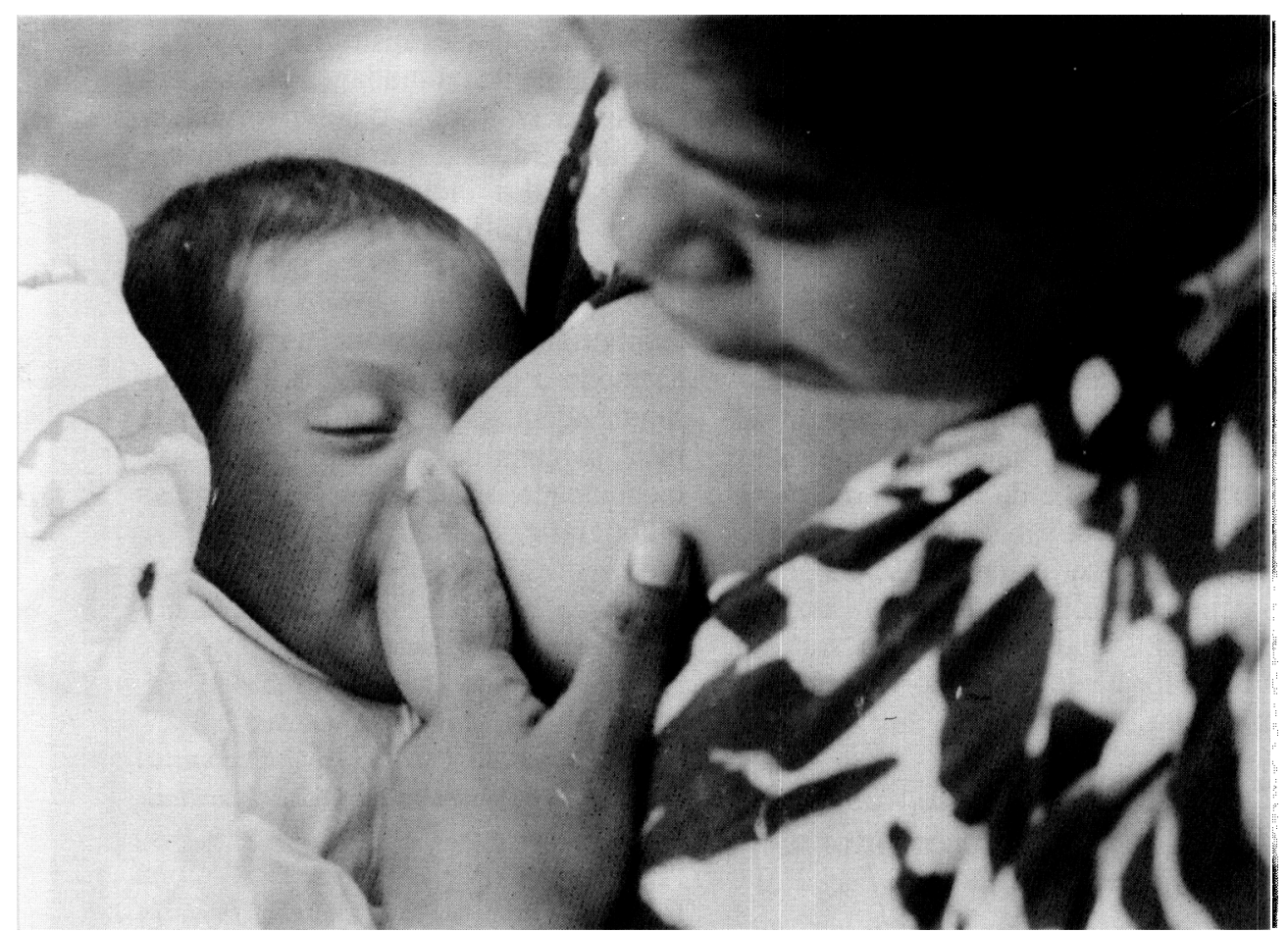

recém-nascido propõe que a criança vem ao mundo com os equipamentos sensorial, motore de comunicação perfeitamente adaptados para a sobrevivência nas condições da espécie. Assim, o melhor desempenho visual do recém-nascido se dá a um foco de aproximadamente 20 $\mathrm{cm}$, a distância usual do rosto da mãc durante a amamentação ao seio, a oportunidade ótima para as trocas sociais necesárias para a formação dos vínculos recíprocos entre mãe e filho, os quais, por sua vez, sãoessenciais para maximizar as chances de sobrevivência da criança, com efeitos críticos sobre todo o seu desenvolvimento. O comportamento do bebê não pode ser compreendido sem referência ao comportamento da mãe; os dois, juntos, constituem um sistema adaptado para a construção de uma relação. Por exemplo, quando a mãe fala com o bebê mantém, geralmente, essa distância de $20 \mathrm{~cm}$, embora essa não seja a melhor distância para o foco visual do adulto.

A evidente orientação social da criança, suas habilidades de reconhecimento individual, principalmente da mãe, bem como suas habilidades comunicativas, reconhecendo e expressando sinais com significado emocional, têm, como consequência relevante, a formação do vínculo, com a construção de parcerias interativas, essenciais para o desenvolvimento humano.

Estudos de acompanhamento (Carlsson e outros, 1978) demonstraram que as mães que tiveram oportunidade de passar mais tempo com o recém-nascido logo após o nascimento, mostraram mais tarde um nível; de responsividade ao bebê mais alto e, o mais importante, um estilo de relacionamento com a criança mais sincronizado, um fator extremamente importante para a construção de uma relação de apego segura. De modo análogo, o mesmo resultado foi observado no estudo com pais (Keller e outros, 1985).

Todos esses conhecimentos devem ser trazidos para a atividade profissional do psicólogo e de outros profissionais ligados ao bebê, como médicos e enfermeiros, embora não se possa imaginar que tal transposição seja direta, mecânica ou mesmo simples.

Recentemente, foram divulgadas informações sobre esse tema através de veículos de massa como jornais e televisão. Em geral, a ênfase dessas informaçð̃es está direcionada para um conceito de capacidades representativas da inteligência da criança.Compreensilvemente, derivam-se interpretações sobre possibilidades de desenvolver a inteligência da 
criança, através de estimulação e treino precoces, até mesmo a partir da vida intra-uterina. Passar de visão de um recém-nascido como uma página em branco, deficiente, indefeso e incompetente para a visão de um mini-gênio pronto para ser desenvolvido a partir da aprendizagem dirigida por um agente externo é permanecer em erro. Os estudos mais recentes sobre as capacidades do bebê absolutamente não autorizam uma visão de que essas habilidades sejam proezas de uma inteligência potencial que cresceria quanto mais fosse estimulada. $\mathrm{O}$ desenvolvimento não é um movimento de soma de aprendizagens induzidas de fora para dentro.

A par de uma melhor compreensão do bebê, os novos conhecimentos de que dispomos fortalecem a idéia do desenvolvimento como construção conjunta entre organismo e ambeinte. Nesse quadro, há, certamente, lugar para a intervenção, no sentido de melhorar a qualidade de vida dos bebês e seus pais e abrir caminhos para um desenvolvimento pleno do ser humano.

Algumas áreas possiveis de atuação vão aqui sugeridas, com a óbvia ressalva de que devem servir apenas como material de reflexão para a construção de práticas profissionais:

\section{Preparação para a materni- dade no período pré-natal}

$\mathrm{O}$ aumento dos conhecimentos e tecnologias méđicas sobre as funçðes de engravidar, parir e criar, bem como o isolamento social dessas funções, têm criado nas mulheres, particularmentenas primiparas, a impressão de que uma pessoa comum não dispõe de conhecimento suficiente para criar um bebê. Além disso, a popularização dos conhecimentos relacionando a conduta da mãe para com o bebê com a saúde mental do futuro adulto trouxe, como consequência colateral, um hiperdimensionamento da responsabilidade das mães na criação. Essas condições, ao lado dos efeitos benéficos com a preocupação com o efeito do ambiente sobre a criança, têm tido um profundo impacto negativo nos sentimentos de segurança necessários para essa atuação. Além disso, a preocupação com o "correto", o "recomendado", o "científico", podem contribuir para deslocar a atenção materna da criança para a instrução de um especialista. Essa é, certamente, uma conduta baseada na noção de que o recém-nascido é um ser passivo, que apenas recebe os cuidados maternos, que apenas recebe o que lhe é

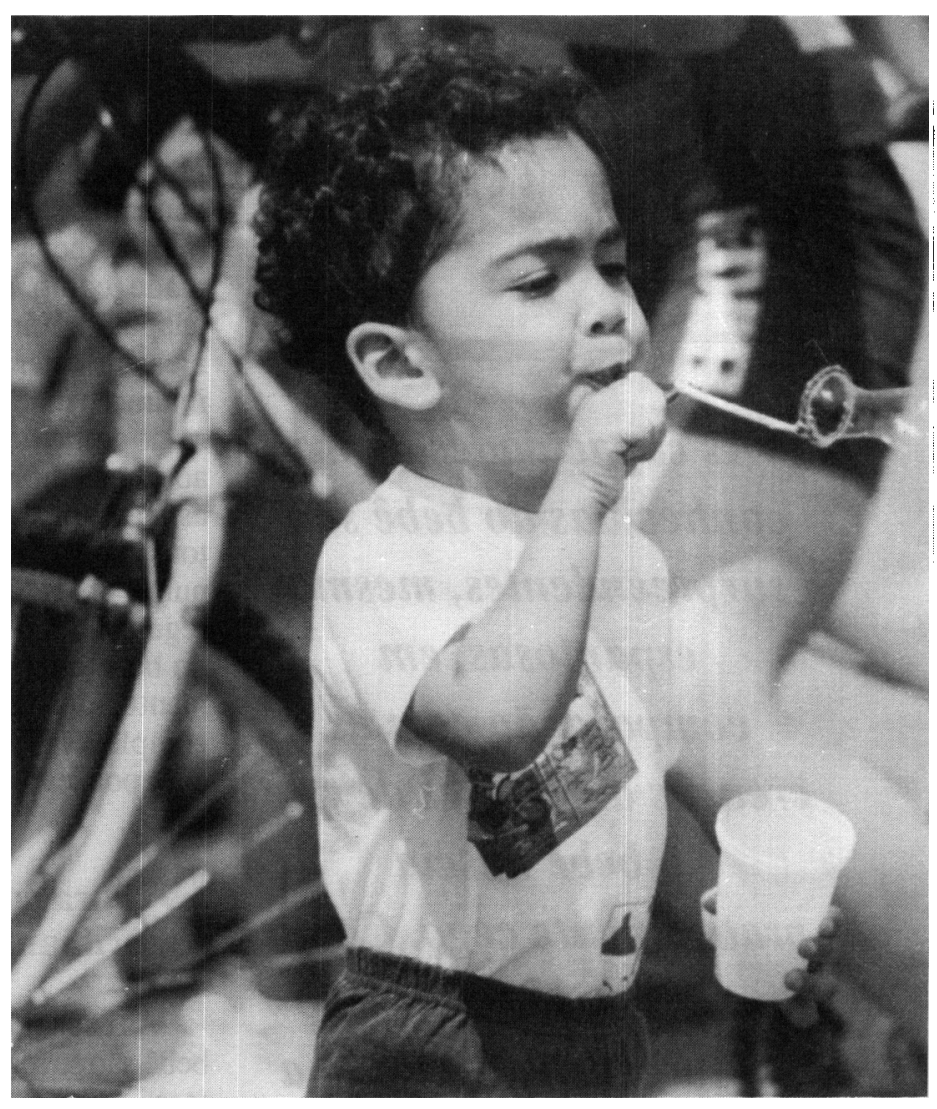

dado. Pensamos que isso é falso e, pior, danoso. Aproveitar os magníficos avanços da ciência que contribuem para a sobrevivência e bem-estar do bebê inclui, com absoluta prioridade, prestar atenção à dupla bebế-mãe, ao que eles desejam, ao que lhes dá prazer e conforto. De modo geral, o bebê e a mãe orientam-se mutuamente no que é melhor para ambos, independentemente de instruções rígidas dos especialistas.

Assim, sugerimos que um item da preparação pré-natal deva centrar-se nessa questão, sem prejuízo do ensino de praticas básicas de puericultura. A redução da ansiedade da mãe em relação à possibilidade de saber cuidar de um bebê talvez possa ser mais fácil se ela puder procurar na própria relação com a crianca indícios que the orientem sobre a melhor forma de cuidar do seu bebê em particular.

\section{2. $O$ uso de instrumentos de avaliação neuro-comporta- mental do bebê}

Desde a década de 70 , novos instrumentos de avaliação de recém-nascidos (prematuros e normais) vêm sendo criados e utilizados, visando, a par dos objetivos ligados ao diagnóstico precoce de dificuldades, o conhecimento das habili- dades do recém-nascido em lidar com os inputs do seu ambiente, enfatizando as diferenças individuais entre os bebês. A importância desses instrumentos reside principalmente na mudança de concepção subjacente sobre o desenvolvimento, admitindo o caráter dinâmico, interacional e individual do processo. Assim, a avaliação neo-natal passa a levar em conta não só os aspectos fisiológicos e neurológicos mas também o comportamento individual na interação, de modo a que os cuidados pós-natais sejam adequados ao "estilo" de cada bebê. Alguns desses instrumentos são mencionados abaixo, com as referências bibliográficas correspondentes.

\section{A. Escala Brazelton de Avaliaçāo Cooportamental Neonatal. \\ - Pessia Grywac Meyerhof (1991). Te- mas sobre o Desenvolvimento. Ano I (2). \\ - Brazelton, T.B. (1984). Neonatal Behavioral Assessment Scale, 2 ed. London: William Heinemann.}

\section{B. Manual for the Assessment of Preterm Infant Behavior (APIB).}

- H. Als, B.M. Lester, E. Z. Tronicke T. B. Brazelton (1982). In Fitzgerald, H.E., Lester, B.M., Yogaman, M.W. (eds) Theory and Research in Behavioral Pediatrics, vol. 1. New York: Plenum Press. 


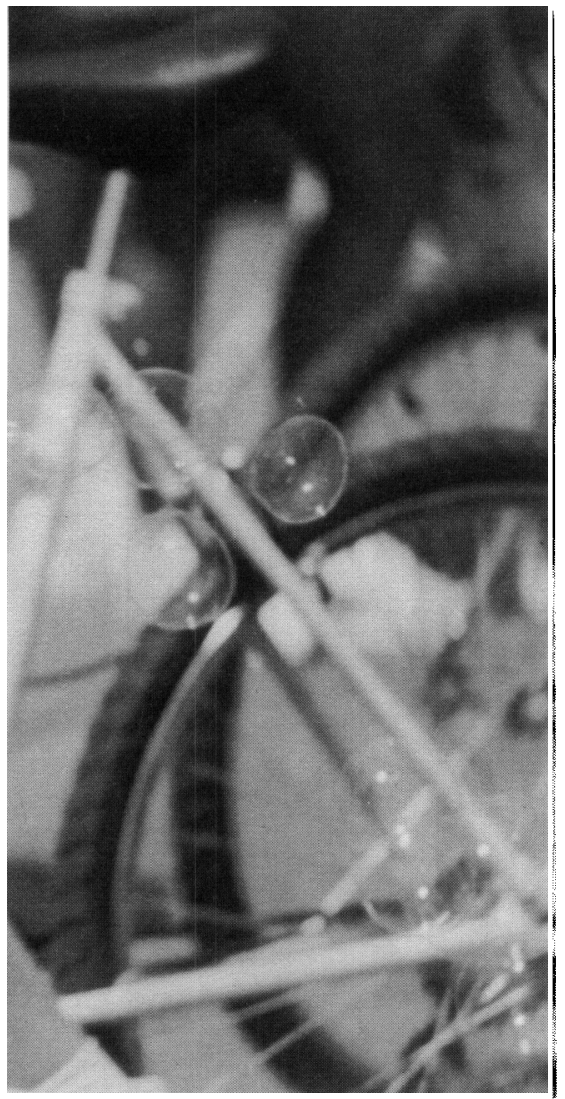

\section{Bibliografia}

C. Manual for the Naturalistic Observation of Newborn Behavior (Preterm and Fullterm Infants)

- Helidelise Als, 1981; 1984 reviosion, The Children Hospital, Boston, Ma, 02115.

Todos esses instrumentos necessitam de treinamento específico para sua aplicação. Eles foram concebidos dentro de um quadro teórico referencial e, como tal, têm sua utilidade restrita a práticas profissionais planejadas de modo abrangente e coerente com a abordagem teórica. Avaliar um recém-nascido com uma escala moderna só tem siginificado dentro de um padrão de atendimento global da parceria bebê-pais, situação em que os resultados da avaliação são usados para melhorar a qualidade de vida da criança e de seus pais.

\section{Aplicação clínica}

As duas aplicaçōes acima citadas dizem respeito a uma intervenção psicoprofilática no atendimento direto à maternidades, creches e ao recém-nascido. No entanto, entendemos que tais conhecimentos sobre a ontogênese podem e devem ser utilizados na formação do corpo teórico que serve de base para a intervenção clínica familiar ou mesmo individual.
ANDRÉ-TOMAS, S. eAUTGAERDEN,S. (1981) Audibilite spontanne de le voix maternelle, audibilite condicionee à Loute autre voix. La Press Medicale, 21

BERGAMASCO, N. H. P. e BERALDO, K.E.A. (1990) "Facial expressions of neonate infants in response to gustatory stimuli". Braz. J. Med. Biol. Res., 23, 245-249.

CARLSSON, S. G.; FAGERBERG, H.; HORNERMAN G.; HWANG, C. LARSSON, A.; RODHOME, M.; SCHAFER J.; DANIELSSON, B. $e$ GUNDEWALL C. (1978) "Effects of varging amounts of contact between mother and child on the mothers nursing behavior". Developmental Psychobiology, 11, 143-150.

CERNOCH,J.M.ePORTER, R.H.(1985) "Recognition of MaternalAxilary Odors by Infants". Child Development, 56, 1593-1598

DECASPERS, A.J.e FIFER W.P.(1980). "Of Human Bonding: Newborns prefer theirmother's voices. Science, 209,11741176.

JOESTER, L. S., PAPOUSEK H. PAPOUSEK, M. (1989) "Patterns of Rhythmic Stimulation by Mothers with Three-Month-Olds: A Cross-Modal Coparison". International Journal of BehavioralDevelopment, 12(2), 143.154.

KUCHUK, A., VIBBERT, M. A. e BORNSTEIN, M.H. (1986) "The Perceptions of Smiling and Its Experimential Correlates in ThreeMonth-OldInfants". ChildDevelopment, 57, 1054-1061.

MASI, W.S. e SCOTT K.G. (1983) "Preterm and Fullterm infants visual responses to mother's and stranger's faces". In Field, T. e Sostek, A. (eds) Infant Born at Risk, cap. 8, 173-179.

MELTZOFFAN., eMOORE,M.K.(1977) "Imitation of facial and manual gestures by human neonates". Science, 198, 75 78 .

MELTZOFF,A.N.eMOOREM.K.(1985) "Cognitive Foundations and Social Funcions of Imitation and Intermodal Representation in Infancy". In Mehler, $J$. \& Fox, R. (eds): Neonate Cognition, cap. 8, p. 139-159.

ECKMAN, P. (1982) Emotions in the Human Face. Cambridge, Univ . Press.

EISENBERG, R.B. (1975) Auditory Competences in the Early Life: The Roots of Communicative Behavior. Baltimore: Univ. Park. Press.

FIELD. T.M. e WALDEN, T. (1982). "Production and Perception of Facial Expressions in Infancy and Early Childhood". In Reese, H. e Lipsitt, L. (eds), Advances in Child Development. Vol. 16. N.Y.: Academic Press.

FIELD, T.M. e outros (1984). "Motherstranger face discrimination by the newborn". Infant Behavior \& Development. Z: 19-25.

GOTTLIEB, G. (1985). "Critical perceptual dimensions of the maternal voice for human infants". In Gottlieb, $G$. \& Krasnegor, N.A. (eds). Measurement of audition and vision, p.21-27.

KELLER, W.D.; HILDEBRANT KA $e$ Richards, M.E (1985) "Effects of Extended Fathers-Infant Contact During the Newborn Period". Infant Behavior and Development, 8, 337-350.

KLAUS M.e KLAUS, P. (1989) O Surpreendente Recém-nascido. Porto Alegre: Artes Médicas.
MONTAGU A. (1988). Tocar - O Significado Humano da Pele .S.P.: Summuns.

NELSON, C.A.(1987). "The recognition of facial expressions on the first two years of life: Mechanisms of development". Child Development, 58 , 889-909.

PAPOUSEK, $H$. $~ P A P O U S E K, M$ (1989). "Ontogeny of Social Interactions in Newborn Infants". In Euler, C. $e$ outros (eds) Neurobiology of Early Infant Behavior, Cap. 19, p. 217-225

THOMANE.B. (1979) "Changing Views of the Being and Becoming of Infants". In thoman, E.(ed): Origins of the Infant Social Responsiveness. New York: John Wiley Sons. Cap. 17, p. 445-459.

TREVARTHENC.(1989) "Development of Early Social Interactions and the Affective Regulation of Brain Growth". In C. euler e outros (eds). Neurobiology of early infant behavior. Cap. 18, p.191216.1

VINTER, A. (1987). A imitação no Recém-Nascido, cap. 1, p. 1-54, S.P.: Manole. 